Hinsichtlich der Kriterien wurden für die Prüfung wissenschaftlich fundierte, geschlechtsunabhängige Systeme der Arbeitsbewertung zugrunde gelegt. ${ }^{19}$ Sie berücksichtigen vier Anforderungsbereiche, die noch einmal in 19 Unterkriterien aufgegliedert sind. Jedes Unterkriterium ist definiert, auch die Anforderungsstufen sind operationalisiert. Die Prüfung mittels Paarvergleich ergibt, dass die Tätigkeiten mindestens gleichwertig sind.

\begin{tabular}{|l|c|c|}
\hline $\begin{array}{l}\text { Anforderungen/Belastungen } \\
\text { (Hauptkriterien) }\end{array}$ & Erzieher/in & $\begin{array}{c}\text { Bautechniker/ } \\
\text { in }\end{array}$ \\
\hline 1. Wissen und Können & 13 & 14 \\
\hline 2. psycho-soziale Anforderungen & 12 & 9 \\
\hline 3. Verantwortung & 4 & 8 \\
\hline 4. Physische Anforderungen & 4 & 0 \\
\hline Summe der Stufenpunkte, ungewichtet & 33 & 31 \\
\hline
\end{tabular}

$\Delta$ Abb. 1: Beispielhafter Paarvergleich zur Feststellung von Gleichwertigkeit (Ergebnisblatt)

\section{Wer kann bzw. muss handeln, um Entgeltgleichheit herzustellen?}

Die derzeitigen Möglichkeiten sind begrenzt: Betroffene Beschäftigte können zwar klagen, jedoch ist eine Einzelklage schwierig, psychisch belastend und zeitlich aufwendig. Personalräte müssen zwar Gleichbehandlung beim Entgelt prüfen, jedoch mangelt es oft an einschlägigem Wissen und Problembewusstsein. Gewerkschaften können zwar Reformkonzepte entwickeln, jedoch müssen sie mehrheitsfähig und in Verhandlungen durchsetzbar sein. Öffentliche Arbeitgeber/innen sind zwar an das Diskriminierungsverbot des AGG gebunden, jedoch ist bislang keine verbindliche Prüfung in Sicht. Der Staat hat sich zwar in Artikel 157 AEUV (Vertrag über die Arbeitsweise der Europäischen Union) zur Sicherstellung des Rechtsprinzips der Entgeltgleichheit verpflichtet, jedoch sind zurzeit keine wirksamen Maßnahmen in Sicht.

19 So insbesondere ABAKABA (Analytische Bewertung von Arbeitstätigkeiten nach Katz und Baitsch, aus der Schweiz) sowie NJC (National Joint Council) aus Großbritannien.

\title{
Weniger Hierarchie und mehr Entgeltgleichheit im Hochschulbereich durch das neue W-Besoldungssystem?
}

\section{Prof. Dr. Sibylle Raasch \\ Universität Hamburg}

Seit sechs Jahren gibt es bundesweit für Hochschulprofessuren ein neues Besoldungssystem „W“. Meine Frage ist, ob diese W-Besoldung mehr Geschlechtergerechtigkeit bei der Vergütung bringen wird.

\section{Die alte C-Besoldung}

Auch unter dem alten Besoldungssystem „C“ gab es Entgeltdifferenzen zwischen Professorinnen und Professoren. Sie beruhten vor allem darauf, dass Frauen nicht nur auf Professuren allgemein unterrepräsentiert waren, sondern ihr Anteil auch innerhalb des 3-stufigen Besoldungssystems (C2 bis C4) der Spitze zu deutlich abnahm: C2 - 18,5 Prozent; C3 13,91 Prozent; C4 - 8,47 Prozent (2004). ${ }^{1}$

Innerhalb der drei Besoldungsstufen wurde nach Alter eingestuft und nach Dienstalter aufgestiegen, so dass sich hier höchstens noch familienbedingte Berufsunterbrechungen zulasten von Frauen auswirken konnten. Weitere Gehaltszuschüsse konnten nur auf C4-Professuren mit dem Ministerium ausgehandelt werden. Sie waren nicht die Regel, kamen wegen der besonderen Frauenunterrepräsentanz auf C4 allerdings eher Männern zugute.

Die gleichstellungspolitischen Gegenstrategien im Hochschulbereich konnten daher den Besoldungsaspekt vernachlässigen. Sie waren darauf gerichtet, mehr Frauen für die Professorenlaufbahn zu gewinnen, den Zugang zu den höhe- ren C-Besoldungsstufen zu verbessern und die Vereinbarkeit von Beruf und Wissenschaftskarriere zu erleichtern. Hier setzen die Hochschulgesetze und hochschulinternen Gleichstellungsrichtlinien an. Hier liegt bislang das Hauptaugenmerk der hochschulinternen Gleichstellungsbeauftragten und der Kontrolle durch die Deutsche Forschungsgemeinschaft (DFG).

Diese Gleichstellungspolitik wirkt: Frauen sind zwar bei den Bewerbungen immer noch unterrepräsentiert. Ihre Berufungsquoten übersteigen seit 2005 jedoch ihren Anteil an den Habilitationen. ${ }^{2}$ Und die Erfolgsquoten der Frauen liegen in den Berufungsverfahren der letzten Jahre leicht über denen der Männer. ${ }^{3}$

\section{Die neue W-Besoldung}

Am 1. Januar 2005 wurde bundesweit das Besoldungssystem „W“ eingeführt. Es gilt für alle neu berufenen Professorinnen und Professoren. Altprofessorinnen und -professoren können in das neue System wechseln. Sie müssen es, wenn bei Bleibeverhandlungen künftig Besoldungserhöhungen er-

\footnotetext{
1 Eigene Berechnungen auf Basis von: Statistisches Bundesamt, Professorlnnenbesoldung B.6.13, Reihe 11, Fachserie 4.4 (Jahrgänge 1998-2006), S. 7

2 Vgl. Dömling, Martina/Schröder, Thomas, Qualitätssicherung in Berufungsverfahren unter Gleichstellungsaspekten. HIS Forum Hochschule 2/2011, Feb. 2011, S. 16.

3 Einschließlich der neuen Juniorprofessuren W1; vgl. Gemeinsame Wissenschaftskonferenz (GWK), Chancengleichheit in Wissenschaft und Forschung, Bonn 2011, S. 11.

4 Vgl. Blomeyer, Christian, Professorenbesoldung, Hamburg 2007, S. 19.
} 


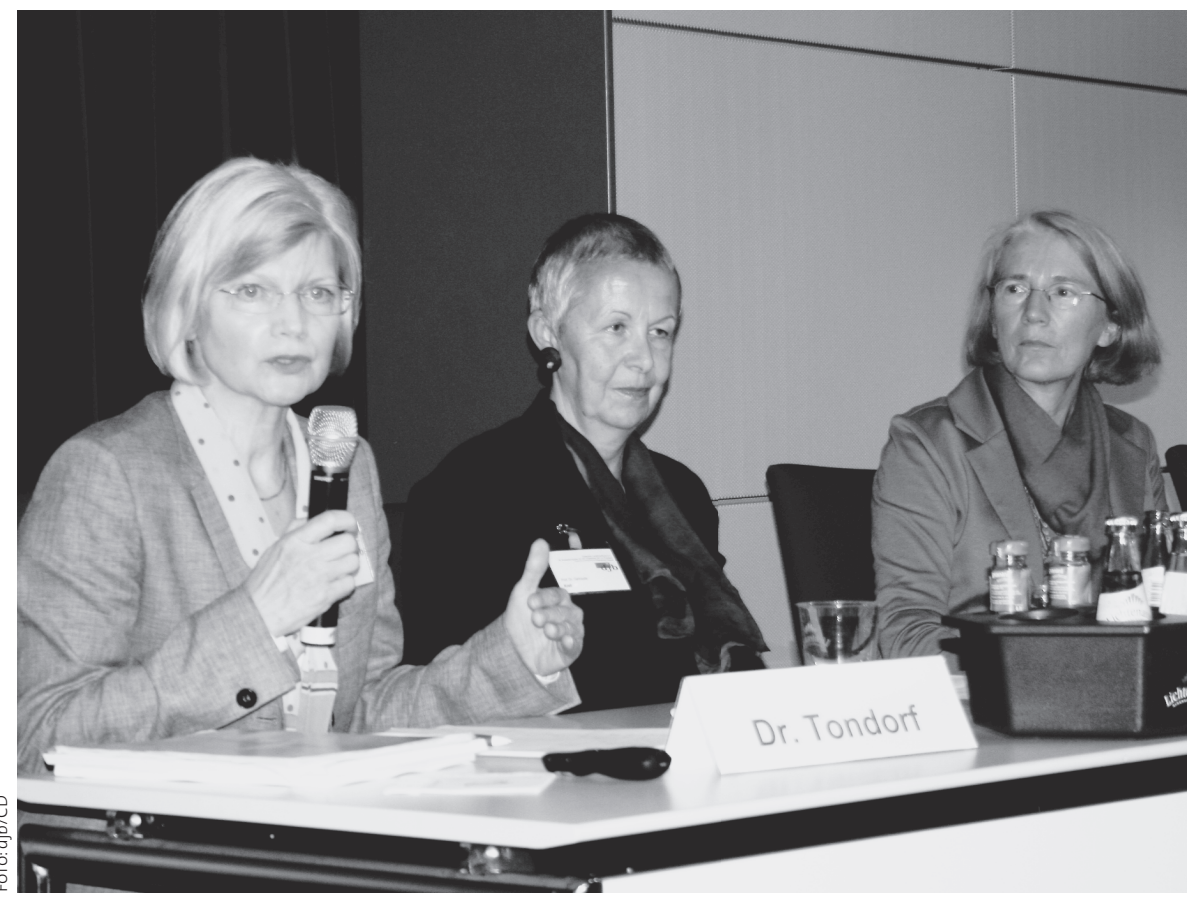

$\Delta$ Dr. Karin Tondorf, Prof. Dr. Gertraude Krell, Dr. Regine Winter (v.I.n.r.) diskutieren das Thema Entgelt(un)gleichheit.

folgen sollen. ${ }^{4}$ Tatsächlich wurde von der Wechseloption bisher wenig Gebrauch gemacht. ${ }^{5}$ Die alte C-Besoldung liegt wegen der bereits erreichten Alterszulagen zumeist höher und bietet über künftige Alterszulagen eine sichere Aussicht auf weiteren Gehaltsanstieg.

Jetzt gibt es nur noch zwei Besoldungsstufen für Professuren: W2 und W3. Vorgeschaltet wurde für die neuen Juniorprofessuren die Stufe W1. Die neuen W-Vergütungen bestehen aus Grundgehalt und variablen Leistungszulagen, letztere allerdings nur für W2 und W3. Alter und Dienstalter spielen keine Rolle mehr. Das Grundgehalt wurde zugunsten größerer Spielräume bei den individuellen Zulagen so niedrig angesetzt, dass vor dem Bundesverfassungsgericht im Oktober 2011 die Frage mündlich verhandelt werden wird, ob diese Besoldung in W2 überhaupt noch amtsangemessen ist. ${ }^{6}$ Einsparung war allerdings kein Ziel der Besoldungsreform. Vielmehr sollte kostenneutral eine stärkere Leistungsorientierung auf Professuren eingeführt und die internationale Wettbewerbsstärke der Hochschulen erhöht werden. ${ }^{7}$ Der Frauenanteil auf allen Stufen von Professuren ist seit der Reform weiter angestiegen, die Unterschiede nach Besoldungsgruppe sind jedoch geblieben: W1 - 37 Prozent; C2 - 20,7 Prozent; W2/C3 - 19,2 Prozent; W3/C4 - 13,6 Prozent (2009). ${ }^{8}$

Wer bestimmt wonach, was auf Professuren eine zulagenfähige Leistung ist? Der Bund machte erste Vorgaben im Bundesbesoldungsgesetz. Doch mit der Föderalismusreform 2006 wurden die Länder für die Hochschulbesoldung zuständig. Diese reichten die konfliktträchtige Konkretisierung der Zulagen an die einzelnen Hochschulen weiter. Und über die Vergabe selbst entscheiden die Hochschulleitungen. Daher bestehen bei der W-Besoldung inzwischen je nach
Bundesland und dort nochmals je nach Hochschule Unterschiede. 70 bis 80 Prozent der Leistungsbezüge entfallen bislang in etwa auf Berufungs- und Bleibe-Leistungsbezüge, 15 bis 20 Prozent auf besondere Leistungsbezüge, der geringe Rest entfällt auf Funktionsleistungsbezüge. ${ }^{9}$ Auch aus Mitteln Dritter können Forschungs- und Lehrzulagen gezahlt werden ( $\mathbb{S} 35$ BBesG), hier eventuell auch an Juniorprofesorinnen und -professoren $(\mathbb{S} 39 \mathrm{Hmb}$ BesG). Weitere Einzelheiten enthalten die Leistungsbezügeverordnungen der Länder und die hochschulinternen Richtlinien über das Verfahren und die Vergabe.

Ruhegehaltsfähig und damit für die Altersversorgung relevant sind Leistungsbezüge, soweit sie unbefristet gewährt und jeweils mindestens zwei Jahre bezogen worden sind ( $\mathbb{S} 3$ Abs. 3 BBesG). Manche Landesgesetze verlangen zudem eine ausdrückliche Erklärung der Ruhegehaltsfähigkeit ( $\mathbb{S} 38$ Abs. $1 \mathrm{HmbBesG}$ ). Auch befristete Leistungsbezüge können nach Verlängerung ( $\$ 33$ Abs. 3 BBesG) oder nach zehnjährigem Bezug ( $\$ 38$ Abs. 1 Satz 1 HmbBesG) für ruhegehaltsfähig erklärt werden. Befristete Funktionszulagen können unter bestimmten Umständen ruhegehaltsfähig werden, beispielsweise nach mindestens fünfjährigem Bezug ( $\int 38$ Abs. 3 HmbBesG). Dabei deckeln alle Gesetze die Höhe der ruhegehaltsfähigen Bezüge im Verhältnis zum einzelnen Grundgehalt und zu den Besoldungsmitteln, die insgesamt zur Verfügung stehen.

Leistungszulagen aus Anlass von Berufungs- und Bleibeverhandlungen bestimmen sich nach individueller Qualifikation, Evaluationsergebnissen, Bewerbungslage und Arbeitsmarktsituation im jeweiligen Fach ( $\mathbb{S}$ HmbHLeistBVO). Leistungszulagen für besondere Leistungen können für Forschung, Lehre, Kunst, Weiterbildung, Nachwuchsförderung und auch das Einwerben von Drittmitteln gewährt werden ( $\$ 4$ Abs. 1 HmbHLeistBVO). Beispielhaft aufgezählt werden in Hamburg unter anderem Forschungs- und Lehrevaluationen, Auszeichnungen, Publikationen, Erfindungen/Patente, wissenschaftliche Redaktion von Fachzeitschriften, wissenschaftliche Arbeitsgruppen, Gutachter- und Vortragstätigkeiten sowie zusätzliche Lehre $(\mathbb{S} 4$ Abs. 3 u. 4 HmbHLeistBVO), wobei die einzelnen Hochschulen hier weiter auswählen und konkretisieren können. Funktionszulagen gibt es in

\footnotetext{
5 Vgl. Blomeyer 2007, S. 50.

6 Vgl. BVerfG, Pressemitteilung 47/2011 v. 21.7.2011.

7 Vgl. Koch, Juliane, Leistungsorientierte Professorenbesoldung, Frankfurt am Main 2010, S. 15

8 Vgl. GWK 2011, Statistischer Anhang Tab. 4.1.2, S. 30.

9 Vgl. Blomeyer 2007, S. 44.
} 
Hamburg nur für die obersten Leitungsebenen, die Präsidien und Dekanate ( $\int 5$ HmbHLeistBVO).

Insgesamt betrachtet bleiben die Vergabekriterien allgemein und die Vergabeverfahren intransparent. Eine Schlüsselstellung haben die Hochschulpräsidien, welche die Berufungsverhandlungen führen und über die Vergabe von Leistungszulagen sowie deren Ruhegehaltsfähigkeit im Einzelfall entscheiden. Und diese Präsidien sind heute noch überwiegend in Männerhand: Rektorinnen/Präsidentinnen - 11,1 Prozent; Prorektorinnen/Vizepräsidentinnen - 23,9 Prozent; Kanzlerinnen - 23,1 Prozent (2010). ${ }^{10}$

\section{Gender Pay Gap?}

Wie wirkt das neue W-System in der Praxis? Empirische Untersuchungen dazu stehen erst am Anfang. Laut HIS-Institut für Hochschulforschung beobachten die Hochschulen bislang überwiegend keine Veränderung der Leistungsorientierung und keine Auswirkungen auf eine Verbesserung der Qualität der Lehre. ${ }^{11}$ Auch eine bundesweite Umfrage des Hochschullehrerbundes (hlb) an Fachhochschulen 2008 brachte keine Hinweise auf eine Steigerung der Leistungsbereitschaft. ${ }^{12}$

Angesichts dieser neuen Leistungszulagen stellt sich jetzt zum ersten Mal ernsthaft die Frage, ob die Besoldung auf Professuren geschlechtergerecht erfolgt oder den bisher schon positionsbedingt vorhandenen Gender Pay Gap in Hochschulen über eine Frauenbenachteiligung bei der Zulagenvergabe sogar noch verstärkt. Die Ergebnisse der hlb-Befragung 2008 wurden dafür einer „equal pay“-Sonderauswertung unterzogen. Dabei zeigte sich, wertete man C- und W-Besoldete zusammen aus, dass Frauen zwar geringfügig schlechtere Erfahrungen als Männer bei der Beantragung individueller Zulagen gemacht hatten (20,7 zu 19,2 Prozent), tatsächlich aber sogar eher Frauen als Männer eine Zulage erhielten (27 zu 23,5 Prozent); wobei die Männer allerdings in der höchsten Gruppe über 1000 Euro leicht dominierten (2,9 zu 3,2 Prozent). ${ }^{13}$

Allein die neuen W-Besoldeten betrachtet ${ }^{14}$, gab es allerdings eine bemerkenswerte Verschiebung: Nur 60 Prozent der Hochschullehrerinnen, aber immerhin 65 Prozent der Hochschullehrer erhalten eine W-Zulage. Und bei den Zulagen 251 bis 500 Euro sowie über 1000 Euro dominieren jetzt die Männer deutlich. Frauen erhalten in der W-Besoldung also seltener und in geringerer Höhe Zulagen als Männer. Allerdings soll dieser Befund nach Berechnungen der Forschungsgruppe statistisch nicht signifikant sein. ${ }^{15}$ Für ein Mehr an Geschlechtergerechtigkeit durch die neue Leistungsbesoldung liefert die Befragung hiermit aber auch keine Anhaltspunkte: Frauen stehen bei Zulagen in der C-Besoldung, wo Zulagen eher selten waren, tendenziell besser da als in der W-Besoldung, wo Zulagen künftig zu einem maßgeblichen Besoldungsbestandteil werden sollen.

Sogar statistisch signifikant sollen festgestellte Differenzen bei der Ruhegehaltsfähigkeit dieser Zulagen sein: Nur 18,5 Prozent der Professorinnen, aber 28,8 Prozent der Pro- fessoren erhalten ruhegehaltsfähige Zulagen. Diese betragen im Durchschnitt bei Professorinnen 470 Euro, bei Professoren aber 563 Euro. ${ }^{16} \mathrm{~W}$-Professorinnen erhalten also seltener und in geringerer Höhe ruhegehaltsfähige Zulagen als WProfessoren - und damit dann später auch eine entsprechend niedrigere Altersversorgung.

Wie erklären sich diese Unterschiede? Da die W-Besoldung erst vor wenigen Jahren eingeführt worden ist, müssen die festgestellten Unterschiede vor allem bei den ersten Berufungs- und Bleibeverhandlungen entstanden sein. Denn erst nach einigen Jahren darf über weitere Zulagen neu verhandelt werden. Die Tatsache, dass Zulagen sofort ruhegehaltsfähig wurden, spricht dafür, dass bei der Ruhegehaltsfähigkeit vor allem geschlechtsspezifische Unterschiede aus der Bezahlung in der vorherigen Tätigkeit in die W-Besoldung weitergereicht wurden. Gerade, wenn von C4 auf W3 gewechselt wird, liegt die Vereinbarung hoher ruhegehaltsfähiger Zulagen nahe. Eine umfassende Erklärung für alle festgestellten oder doch möglichen Unterschiede bei der Zulagenvergabe ist dies aber nicht.

Leo Hellemacher kann durch weitere Berechnungen ausschließen, dass ein unterschiedliches Alter und damit unterschiedliche Berufserfahrung bei Frauen und Männern bzw. die unterschiedliche Besoldungshöhe in einzelnen Bundesländern oder die besondere finanzielle Förderung bestimmter Fächer für die Unterschiede bei der Zulagenvergabe verantwortlich sind. ${ }^{17}$ Diese Unterschiede andererseits bloß durch weniger Verhandlungsgeschick und schlechteres Self-Marketing der Professorinnen zu erklären, dürfte angesichts ihres doch schon höheren Lebensalters bei der Berufung auf eine Erstprofessur und angesichts der sehr hohen Qualifikationsanforderungen zu kurz gegriffen sein. ${ }^{18}$ Über die weiteren Ursachen können wir gegenwärtig allerdings nur Vermutungen anstellen.

Hellemacher erinnert an Forschungsergebnisse, dass Frauen eher auf Arbeitsinhalte als auf Geld und Aufstieg achten würden, dass Frauen anders als Männer neben dem finanziellen Kalkül auch Überlegungen zur künftigen Vereinbarkeit von Beruf und Familie anstellten. Frauen seien daher im Finanziellen kompromissbereiter. Und da die Hochschulen in-

10 Vgl. GWK 2011, S. 12.

11 Jaeger, Michael/In der Smitten, Susanne, Leistungsbezüge in der WBesoldung. HIS: Forum Hochschule 16/2011, Hannover 2011, S. 10.

12 Vgl. Hellemacher, Leo/Stelzer-Rothe, Thomas, Ergebnisse einer bundesweiten Befragung der öffentlich-rechtlichen Fachhochschulen im Jahre 2008, Die Neue Hochschule (DNH) 1/2009 S. 8 ff., S. 12.

13 Vgl. Simons, Katrin/Hellemacher, Leo, W-Zulagen und Entgeltgleichheit an Hochschulen, in: DNH 4-5/2009 S. $14 \mathrm{f}$.

14 Vgl. Simons/Hellemacher 2009, S. 14; Hellemacher, Leo, Gender-PayGaps an Hochschulen, DNH 3/2011 S. 122 ff., S. 122 f.

15 Das bedeutet, dass für die Unterschiede zwischen den Messgrößen die Wahrscheinlichkeit nicht gering ist, dass sie durch bloßen Zufall zustande gekommen sind. Dafür gibt es statistische Berechnungsformeln. Umgekehrt kann aber auch nicht mit Sicherheit geschlossen werden, dass bei einem statistisch nicht signifikanten Ergebnis tatsächlich kein Unterschied vorliegt.

16 Vgl. Simons/Hellemacher 2009, S. 15; Hellemacher 2011, S. 123.

17 Vgl. Hellemacher 2011, S. $122 \mathrm{ff}$.

18 Ebenso Hellmacher 2011, S. 124. 
zwischen unternehmerisch ausgerichtet würden, werde auf diese Kompromissbereitschaft der Frauen vermutlich nur allzu gern eingegangen. ${ }^{19}$

Noch ernster nehmen möchte ich Hellemachers Vermutung ${ }^{20}$, dass bei den Berufungs- und Bleibeverhandlungen allzu leicht für Frauen nachteilige Vorurteile und Rollenklischees zum Tragen kommen könnten. Die jüngste HIS-Untersuchung von Berufungsverfahren an niedersächsischen Hochschulen $^{21}$ identifiziert Berufungsverfahren als letzte „Black Box“ und zugleich letztes Refugium der Präsidien. Da hier das meiste nur unter vier oder sechs Augen geklärt werde, seien Bewerber/innen dabei recht ungefiltert subjektiven und vor allem geschlechtsspezifischen Einschätzungen und Beurteilungen ausgesetzt. Dahinter stünden im Regelfall keine bewussten Diskriminierungsstrategien, sondern alltägliche Wahrnehmungsweisen, die eine geschlechtsspezifisch unterschiedliche Qualität der Ausfüllung von Berufsrollen unterstellten. Insofern müssen aus Frauensicht wenn schon nicht eine Teilnahme der Gleichstellungsbeauftragten an den Berufungsverhandlungen, so doch zumindest objektivierte Leistungsstandards und maximale Transparenz bei der Vergabe von Leistungszulagen gefordert werden.

Hier sehe ich ein weiteres Problem. Die Standards der heutigen Besoldungsregelungen zur Bemessung wissenschaftlicher Leistung geben zwar Objektivität, Kriterienvielfalt und Geschlechtsneutralität vor. Gerade bei Berufungsverhandlungen setzen die Hochschulpräsidien inzwischen jedoch zunehmend auf das Einwerben von Drittmitteln, während sonstige Forschung, die Qualität der Lehre, die persönliche Betreuung Studierender und das Engagement in der akademischen Selbstverwaltung in den Hintergrund treten. Da die meisten Hochschulen unterfinanziert sind, werden sie auch von der Politik immer stärker in die Drittmitteleinwerbung gedrängt. Damit rücken aber Tätigkeitssegmente von Professuren in den Hintergrund der Berufungsverhandlungen, welche für die Funktionsfähigkeit der Hochschulen ebenfalls unerlässlich sind und in welche nach meinen Beobachtungen gerade Frauen besonders viel und oft mehr als Männer investieren. Insofern dürfte sich die immer stärkere Fixierung der Hochschulleitungen auf Drittmitteleinwerbung auf die Hochschulentwicklung allgemein und die Entgeltgleichheit im Besonderen nachteilig auswirken.

Auch an der Universität Hamburg zählen vor allem Drittmittel. Dabei zeigte ein erster Blick in die Akten, dass Frauen lediglich 86 Prozent dessen bekommen, was Männer an befristeten Berufungs- und Bleibezulagen heraushandeln. Bezieht man die Höhe der von den Frauen zugesagten Drittmittel mit ein, zeigt sich allerdings, dass die Frauen nur 43 Prozent dessen zugesagt haben, was die Männer versprachen. Insofern hatten diese Professorinnen eigentlich sogar noch gut verhandelt; vielleicht weil an der Universität Hamburg für ein paar Jahre eine Präsidentin amtierte, die Kanzlerin eine Frau ist und es heute eine Vizepräsidentin für die Berufungen gibt?
Marktwert und Leistung werden also zunehmend gemessen an den eingeworbenen Drittmitteln, daneben auch an der Zahl von Publikationen oder Zitationen in internationalen referierten Fachzeitschriften nach dem Science Citation Index. Das erscheint auf den ersten Blick ein Fortschritt, weil diese Kriterien objektiv und geschlechtsneutral sind. Hildegard Matthies und Karin Zimmermann, langjährig erfahren im Bereich der Wissenschafts- und Geschlechterforschung, warnen aber, dass ein so definierter Wettbewerb weder transparent noch frei von Wettbewerbsverzerrung sei. ${ }^{22}$ Die tendenziell zu beobachtende Abwertung der Leistungen von Frauen schlage sich auch auf die Häufigkeit der Zitationen ihrer Arbeiten nieder. Die Begutachtung von Forschungsanträgen im Peer-Review-Verfahren führe erwiesenermaßen dazu, dass Antragstellende mit hoher Reputation positiver beurteilt würden und sich nicht unbedingt die besten Forschungsanträge durchsetzten. Die Chance auf Bewilligung von Forschungsgeldern steige mit der Summe bereits empfangener Forschungsgelder. Demnach weise auch die Vergabe von Forschungsmitteln einen Geschlechterbias auf. Sind die Hamburger Professorinnen, die weniger Drittmittel versprechen als die Männer, also vielleicht einfach Realistinnen trotz gleicher Qualifikation?

Die ersten empirischen Befunde zur neuen W-Besoldung werfen also mehr Fragen zur geschlechtergerechten Besoldung im Hochschulbereich auf, als sie beantworten. Für die Gleichstellungspolitik bedeutet das, hier weiter zu forschen und zumindest auf größere Transparenz bei der Vergabe von Leistungszulagen zu drängen. Objektive Entscheidungsmaßstäbe sind erst einmal ein Fortschritt, wenn damit keine Verengung auf bloße Drittmitteleinwerbung einhergeht. Doch jedes Kriterium ist gleich wieder auf seine geschlechterpolitischen Implikationen und Auswirkungen zu hinterfragen. Die Gleichstellungsbeauftragten an den Hochschulen und die Genderforscherinnen und -forscher sollten die weitere Entwicklung der W-Besoldung unter dem Gesichtspunkt einer Verstärkung des bereits vorhandenen Gender Pay Gaps scharf im Auge behalten.

\footnotetext{
19 Vgl. Hellemacher 2011, S. 124.

20 Vgl. Hellemacher 2011, S. 125.

21 Vgl. Dömling, Martina/Schröder, Thomas, Qualitätssicherung in Berufungsverfahren unter Gleichstellungsaspekten, HIS: Forum Hochschule 2/2011, Hannover Feb. 2011, S. 38 f.

22 Vgl. Matthies, Hildegard/Zimmermann, Karin, Arbeitsfeld Wissenschaft und Geschlechtersegregation, WSI Mitteilungen 5/2011, S. 265 ff., S. 267.
} 